\title{
GENERAL SOLUTION OF THE PROBLEM OF ELASTOSTATICS OF AN $n$-DIMENSIONAL HOMOGENEOUS ISOTROPIC SOLID IN AN $n$-DIMENSIONAL SPACE
}

\author{
BY H. M. WESTERGAARD
}

1. Introduction. Dealing with the important case of a threedimensional solid subject to constant body forces (such as gravity) B. Galerkin* expressed the stresses and the displacements in terms of three functions, governed by the fourthorder equation $\Delta \Delta f=$ const., and mutually independent except through the boundary conditions. He has demonstrated the fruitfulness of his method in later papers. $\dagger$

It is profitable to interpret Galerkin's three functions as components of a vector. Simplicity is gained and significance is added by doing this. It is proposed to call this vector the Galerkin vector. Its nature is such that only a slight amount of complexity is added in the general derivations by considering an $n$-dimensional space.

2. Notation. Let the following notation be used.

$i_{1}, i_{2}, \cdots, i_{m}, \cdots, i_{p}, \cdots, i_{n}=$ unit vectors in $n$ directions perpendicular to one another; $m \neq p$.

$$
R=i_{1} x_{1}+i_{2} x_{2}+\cdots+i_{n} x_{n}=\text { radius vector drawn from }
$$
the origin to any point; the point is called point $R$.

$$
\boldsymbol{\varrho}=i_{1} \xi_{1}+i_{2} \xi_{2}+\cdots+i_{n} \xi_{n}=\text { displacement }=\text { increment of }
$$

$\boldsymbol{R}$. The point $\boldsymbol{R}$ moves to the position $\boldsymbol{R}+\boldsymbol{\varrho} ; \boldsymbol{\varrho}$ is assumed small.

$$
\boldsymbol{P}=i_{1} P_{1}+i_{2} P_{2}+\cdots+i_{n} P_{n}=\text { force. }
$$

$K=i_{1} K_{1}+i_{2} K_{2}+\cdots+i_{n} K_{n}=$ body force which is distrib-

* B. Galerkin, Contribution à la solution gênérale du problème de la thêorie de l'élasticité dans le cas de trois dimensions, Comptes Rendus, vol. 190 (1930), p. 1047; Contribution a l'investigation des tensions et des déformations d'un corps élastique isotrope (in Russian), Comptes Rendus de l'Académie des Sciences de l'URSS, (1930), p. 353.

† Comptes Rendus, vol. 193 (1931), p. 568; vol. 194 (1932), p. 1440; vol. 195 (1932), p. 858; and papers in Russian: Comptes Rendus de l'Académie des Sciences de l'URSS, (1931), p. 273 and p. 281; Messenger of Mechanics and Applied Mathematics, Leningrad, vol. 1 (1931), p. 49; Transactions of the Scientific Research Institute of Hydrotechnics, vol. 10 (1933), p. 5. 
uted through the solid, per unit of magnitude of the region considered; of the dimension force times distance ${ }^{-n} ;$ a function of $R$.

$S_{m}=$ section defined by $x_{m}=$ const. The section is called a front face if the part of the solid dealt with lies on the side of smaller values of $x_{m}$; and a back face if the part dealt with lies on the side of greater values of $x_{m}$.

$\mathrm{s}_{m}=$ vector representing internal force or stress on a small region of the front face $S_{m}$ at point $R$, per unit of magnitude of this region; of the dimension force times distance ${ }^{-n+1}$; and $-s_{m}=$ stress on the same region of the back face $S_{m}$.

$\sigma_{m}=$ component of $s_{m}$ in the direction $i_{m}$; normal stress in the direction of $i_{m}$.

$\tau_{m p}=$ component of $s_{m}$ in the direction of $i_{p},(p \neq m)$; shearing stress on the front face $S_{m}$ in the direction $i_{p}$.

$\Theta=\sigma_{1}+\sigma_{2}+\cdots+\sigma_{n}=$ bulk stress.

$E=$ Young's modulus of elasticity (equation (6)).

$G=$ modulus of elasticity in shear (equation (7)).

$\mu=$ Poisson's ratio of lateral contraction (equation (6)).

$F=i_{1} X_{1}+i_{2} X_{2}+\cdots+i_{n} X_{n}=$ Galerkin vector .

$$
\nabla=i_{1} \frac{\partial}{\partial x_{1}}+i_{2} \frac{\partial}{\partial x_{2}}+\cdots+i_{n} \frac{\partial}{\partial x_{n}} .
$$

$$
\begin{aligned}
\operatorname{div} F & =\frac{\partial X_{1}}{\partial x_{1}}+\frac{\partial X_{2}}{\partial x_{2}}+\cdots+\frac{\partial X_{n}}{\partial x_{n}} . \\
\Delta & =\operatorname{div} \nabla=\frac{\partial^{2}}{\partial x_{1}^{2}}+\frac{\partial^{2}}{\partial x_{2}^{2}}+\cdots+\frac{\partial^{2}}{\partial x_{n}^{2}} .
\end{aligned}
$$

3. Equilibrium. A set of forces $\boldsymbol{P}$ applied at the points $\boldsymbol{R}$ is in equilibrium when the $n$ conditions

$$
\sum P_{m}=0,
$$

and the $n(n-1) / 2$ conditions

$$
\sum P_{m}\left(x_{p}-C_{p}\right)=\sum P_{p}\left(x_{m}-C_{m}\right)
$$

are satisfied, each sum including all the force components in the single direction of either $i_{m}$ or $i_{p}, C_{m}$ and $C_{p}$ being constants which are interpreted as coordinates in the directions of $i_{m}$ and $i_{p}$ of an arbitrary fixed point $C$.

Consider an element of the solid bounded by back faces $S_{1}, S_{2}, \cdots, S_{n}$ intersecting at the point $R$, and by front faces 
$S_{1}, S_{2}, \cdots, S_{n}$ intersecting at the point $R+d R$. With all the forces included which act on this element, and with $C$ at the center of the element, equation (2) leads to the $n(n-1) / 2$ conditions

$$
\tau_{m p}=\tau_{p m} .
$$

Equation (1) leads to the $n$ conditions

$$
\frac{\partial \sigma_{m}}{\partial x_{m}}+\sum_{\substack{1,2, \cdots, n \\ \text { excl. } m}}^{p} \frac{\partial \tau_{p m}}{\partial x_{p}}+K_{m}=0
$$

Because of equation (3), equation (4) may be rewritten in the form

$$
\operatorname{div} s_{m}+K_{m}=0 .
$$

4. Elasticity. Hooke's law of stresses and deformations is stated in the following form. The strain in the direction of $i_{m}$ is

$$
\frac{\partial \xi_{m}}{\partial x_{m}}=\frac{1+\mu}{E} \sigma_{m}-\frac{\mu}{E} \Theta,
$$

and the detrusion in the directions of $i_{m}$ and $i_{p}$ is

$$
\frac{\partial \xi_{m}}{\partial x_{p}}+\frac{\partial \xi_{p}}{\partial x_{m}}=\frac{\tau_{m p}}{G} .
$$

With the constants $E$ and $\mu$ given, only one value of $G$ leads to isotropy. The value

$$
G=\frac{E}{2(1+\mu)}
$$

represents isotropy in the cases $n=2$ and $n=3$, and is assigned to $G$ here. That this value actually represents isotropy for any value of $n$ is concluded from the form of equation (13), which is derived from equations (5) to (8). Equation (6) gives

$$
\operatorname{div} \varrho=\frac{1-(n-1) \mu}{E} \Theta,
$$

which, with (8), permits the rewriting of (6) in the form

$$
\sigma_{m}=2 G\left(\frac{\partial \xi_{m}}{\partial x_{m}}+\frac{\mu}{1-(n-1) \mu} \operatorname{div} \varrho\right) \text {. }
$$


Equations (7) and (10) lead to the following formula for the resultant stress on the front face $S_{m}$ :

$$
s_{m}=G\left(\nabla \xi_{m}+\frac{\partial \varrho}{\partial x_{m}}\right)+i_{m} \frac{2 G \mu}{1-(n-1) \mu} \operatorname{div} \mathbf{o} .
$$

Substitution from equation (11) in equation (5) gives

$$
\Delta \xi_{m}+\frac{1-(n-3) \mu}{1-(n-1) \mu} \frac{\partial}{\partial x_{m}} \operatorname{div} \mathbf{\varrho}+\frac{K_{m}}{G}=0,
$$

or, in vector form,

$$
\left(\Delta+\frac{1-(n-3) \mu}{1-(n-1) \mu} \nabla \operatorname{div}\right) \varrho+\frac{K}{G}=0 .
$$

Isotropy is attained because equation (13) is independent of the orientation of the axes of coordinates.

5. The Galerkin Vector. The general solution of equation (13) is the general solution of the problem. A difficulty arises from the interdependence of the components. This difficulty is overcome by introducing the Galerkin vector $F$.

The displacement is expressed as

$$
\mathbf{\varrho}=\frac{1}{2 G}(c \Delta-\nabla \operatorname{div}) F
$$

in which $c$ is a constant yet to be selected. By substituting $\varrho$ from equation (14) in equation (13) and noting that

$$
\Delta \nabla \operatorname{div}=\nabla \operatorname{div} \Delta=\nabla \operatorname{div} \nabla \operatorname{div},
$$

it is found that the terms containing the combined operators shown in equations (15) disappear when

$$
c=\frac{2(1-(n-2) \mu)}{1-(n-3) \mu} .
$$

Then equations (14) and (13) become

$$
\begin{aligned}
\boldsymbol{\varrho} & =\frac{1}{2 G}\left[\frac{2(1-(n-2) \mu)}{1-(n-3) \mu} \Delta-\nabla \operatorname{div}\right] F, \\
\Delta^{2} F & =-\frac{1-(n-3) \mu}{1-(n-2) \mu} K .
\end{aligned}
$$


The general solution of equation (18) defines the general solution for 0 through equation (17).

6. Stresses. The vector $F$ and its components $X_{1}, X_{2}, \cdots, X_{n}$ lend themselves to expressions for the stresses. Equation (17) gives

$$
\xi_{m}=\frac{1}{2 G}\left[\frac{2(1-(n-2) \mu)}{1-(n-3) \mu} \Delta X_{m}-\frac{\partial}{\partial x_{m}} \operatorname{div} F\right]
$$

and

(20) $\quad \operatorname{div} \varrho=\frac{1-(n-1) \mu}{2(1-(n-3) \mu) G} \Delta \operatorname{div} F$.

Substitution from equations (19) and (20) in equations (10), (7), (9), and (11) leads to the formulas

$$
\sigma_{m}=\frac{2(1-(n-2) \mu)}{1-(n-3) \mu} \frac{\partial \Delta X_{m}}{\partial x_{m}}
$$

$$
+\left[\frac{\mu}{1-(n-3) \mu} \Delta-\frac{\partial^{2}}{\partial x_{m}{ }^{2}}\right] \operatorname{div} F,
$$

$$
\begin{aligned}
\tau_{m p} & =\frac{1-(n-2) \mu}{1-(n-3) \mu}\left[\frac{\partial \Delta X_{m}}{\partial x_{p}}+\frac{\partial \Delta X_{p}}{\partial x_{m}}\right]-\frac{\partial^{2}}{\partial x_{m} \partial x_{p}} \operatorname{div} F \\
\Theta & =\frac{1+\mu}{1-(n-3) \mu} \Delta \operatorname{div} F
\end{aligned}
$$

and

$$
\begin{aligned}
\mathbf{s}_{m}=\frac{1-(n-2) \mu}{1-(n-3) \mu} & {\left[\nabla \Delta X_{m}+\frac{\partial}{\partial x_{m}} \Delta F\right] } \\
+ & {\left[\frac{i_{m} \mu}{1-(n-3) \mu} \Delta-\frac{\partial}{\partial x_{m}} \nabla\right] \operatorname{div} F . }
\end{aligned}
$$

The form of equations (17) and (18) shows that $F$ is independent of the orientation of the axes of coordinates. It follows that equations (19) to (24) continue to apply with the same $F$ after a re-orientation of the axes of coordinates.

UNIVERSITY OF ILLINOIS 